\title{
Short Term Effect of Acupuncture in Chronic Low Back Pain: A Case Study
}

\author{
Supriya T Kalle* \\ Physiotherapy, Coventry University, UK
}

Submission: November 28, 2017; Published: January 05, 2018

*Corresponding author: Supriya T Kale, Physiotherapy, Coventry University, London, UK, Email: supriyakale18@gmail.com

Abstract

A 16-year old girl presented to a NHS clinical setting with a complaint of sporadic low back pain (LBP), which she had been experiencing since the age of 13. The LBP had been worsening over the period of the preceding two months. The previous treatment involved only painkillers. The patient had never had acupuncture or physiotherapy treatment for her LBP and was sceptical about its effect. The aggravating factors involved prolonged standing, bending, and lifting. The easing factors were painkillers and rest. The pain was affecting her part-time job. The patient was treated with 3 sessions of acupuncture over 7 weeks. Acupuncture was used with the aim of reducing pain and improving the lumbar spine range of motion. Though the sessions were not frequent, acupuncture was successful in achieving pain reduction. This case report shows the short-term effect of the acupuncture as a treatment in a case of chronic LBP.

Keywords: Lower back pain (LBP); Acupuncture; Sporadic pain; Acupuncture sessions

\section{Introduction}

Low back pain (LBP) is one of the most common musculoskeletal disorders. The data collected by a National Ambulatory Medical Care Survey (NAMCS) suggests that 2.3\% of visits to the physician are attributed to LBP, which has changed little since 1990s [1]. If serious spinal pathologies are excluded, most of the cases presented in the primary care are non-specific LBP [2]. It is one of the main causes of disability in working adults, even though it responds to a wide spectrum of interventions $[3,4]$. In UK, the annual total cost of LBP to the economy is estimated to be $£ 12.3$ billion [5]. According to the NICE guidelines, one third of the UK's population is affected by LBP [6].

Various evidence suggest better outcome measures after classifying the patient in one or more of the five dominant movement-based classifications, but these classifications are less frequently used in the clinical practice [7]. Witt et al. [8] identified some characteristics that indicate better responses to acupuncture i.e. young age, education of more than 10 years, and worse back baseline function, whereas patients with gradual onset may respond favourably [9]. In a recent comparative study by Oakes \& Bassett [10], it was found that individualized targeted acupuncture and physiotherapy showed better outcomes than group acupuncture and physiotherapy in 51 patients with low back pain. The author did recommend using the Four Gates treatment, if it helps in easing the pain at later stages [10]. There are various clinical prediction rules that indicate the efficacy of spinal manipulation, lumbar stabilization exercises, and facet joint block as treatments for LBP, but there is a scarcity of good evidence indicating the efficacy of acupuncture as a treatment for LBP [11-13].

Previous research, focusing on the use of acupuncture in the treatment of LBP, is conflicting and many trials have poor methodology. Due to the conflict regarding the efficacy of acupuncture for the treatment of LBP, acupuncture is not used as a treatment for LBP in many clinical settings. The other reason may also be that patients have found conventional medical treatment to be unsatisfactory [14]. But since the National institute of clinical Excellence (NICE) guidelines of 2009, acupuncture has been popular among clinicians and patients of chronic LBP. According to Manheimer et al. [15], the current data indicates that acupuncture is more beneficial than sham acupuncture and no additional treatment for short-term relief for chronic LBP. However, in spite of these positive results, due 
to lack of quality long-term data, it is difficult to predict the longterm effect on the condition. The following case report shows the efficacy of acupuncture, in conjunction with other physiotherapy interventions, in a young patient with chronic LBP.

\section{Case Report}

\section{Subjective examination}

The patient of the present case study was a young girl of 16 years of age. She complained of localised central low back pain, which occasionally referred to either the left or right side of the lower back, her Visual Analogue scale (VAS) was 5-9/10. The nature of the pain was a constant bruise-like feeling with no neurological signs or symptoms. Prolonged standing, bending, and lifting aggravated the symptoms, whereas painkillers and rest were helpful in easing the symptoms. These factors suggested the presence of a mechanical component within the patient's problem [16]. Turning in bed occasionally caused sleep disturbance, but the patient was able to fall back to sleep soon after getting into a comfortable position The Patient had a history of sporadic LBP, since the age of 13 , and the previous treatment involved only painkillers. The pain was worse at the time of the examination, and there had been so for the preceding two months. It had a gradual onset and no investigation had been done until the patient presented herself to the clinic. The pain worsened every morning and continued during the day, and thus was gradually worsening. Pain was exacerbated by sneezing, but not by coughing. The patient reported feelings of tiredness due to the LBP. She was screened for red and yellow flags, and none were found. She was a college student, and worked part-time as a retailer in a shop, which involved prolonged standing. As this is one of the aggravating factors, patient was finding it difficult to work. She also occasionally went out with friends, but was not involved in any sports.

\section{Objective examination}

There were no postural abnormalities detected while walking, standing, or sitting. The lumbar spine active range of motion (AROM) was painful and reduced by $50 \%$ in the flexion and the bilateral side flexion. There was no pelvic mal-alignment while standing or during lumbar spine AROM. She had full passive range of motion (PROM) in her hip joints, but flexion Table 1: Treatment schedule. and rotation produced the symptoms bilaterally. Tests, such as Patrick's test, thigh thrust test, and FABER test produced the symptoms but no hip joint pain was evident on palpation. When performing the sacroiliac joint (SIJ) tests like iliac compression and distraction, the sacrum thrust produced similar symptoms on the right side of the lower back. There was mild tenderness and tightness in the right gluteal muscles and piriformis muscle. There was weakness in the core muscles but no paraspinal muscle spasm was found. Straight leg raise (SLR) was normal bilaterally. Since there were no neurological signs or symptoms, no neurological examination was carried out.

Observations from the three tests, along with pain on the lumbosacral segment, suggested SIJ dysfunction [17-19] but since the patient was already in pain, it was difficult to establish a diagnosis. Palpation of the lumbar spine showed tenderness and stiffness on the lower lumbar spine and bilateral SIJ. The inter-examiner reliability of PAIVMS on the lumbar spine has been shown to be poor to fair, and most of the available evidence is of poor methodology [20].

Based on the complete subjective and objective examination, the physiotherapist hypothesised 2 diagnoses: mechanical LBP or SIJ dysfunction. The proposed treatment plan was discussed with the patient: ergonomic advice and education, manual therapy, exercise therapy, and acupuncture if required. The Home Exercise Programme (HEP), involving core strengthening exercises, ergonomic advice, and education and advice to use heat or cold over the painful areas was given to the patient at the end of the session. It was explained to the patient that further reassessment could clarify the diagnosis. The reassessment during a follow up appointment will assist the physiotherapist in finalising a diagnosis.

\section{Treatment}

Acupuncture was started from the second follow-up session, after the failure in achieving any subjective improvement in the patient's symptoms post hands-on treatment i.e. manual therapy. As the patient was seen in an NHS clinical setting, the patient was allowed only 6 physiotherapy sessions in her first referral hence 3 sessions of acupuncture were provided over a period of 7 weeks. The treatment schedule is outlined in Table 1.

\begin{tabular}{|c|c|c|c|c|c|}
\hline Sessions & Acupuncture Points & Duration (minutes) & Needle Size & Depth of Insertion (cun) & De qi \\
\hline Session 1 & BL 23, 24, 25 & 20 minutes & $0.25 / 40 \mathrm{~mm}$ & 1.5 - $2 \mathrm{~cm}$ depth & Achieved- mild \\
\hline Session 2 & BL 23, 24, 25 3 HJJ & 20 minutes & $0.25 / 40 \mathrm{~mm}$ & $1.5-2 \mathrm{~cm}$ depth & Achieved- strong \\
\hline Session 3 & BL 23,24,25 3 HJJ, LI4 & 20 minutes & $\begin{array}{l}0.25 / 40 \mathrm{~mm} \\
0.25 / 25 \mathrm{~mm}\end{array}$ & $\begin{array}{l}1.5-2 \mathrm{~cm} \text { depth } \\
0.5-1 \mathrm{~cm} \text { depth }\end{array}$ & Achieved- mild strong \\
\hline
\end{tabular}


The patient had no previous experience of acupuncture hence she was slightly sceptical about its efficacy. Although the patient was unsure about its effectiveness, she wished to proceed as she wanted pain relief. The entire procedure and normal sensations experienced during and post treatment were explained and discussed with the patient. All the risks and contraindications were discussed and taken into consideration. A consent form was signed by the patient and the guardian (her grandmother) before initiating the treatment.

The patient's position for all the treatment sessions was prone, exposing the lower back area. The needles used were Classic Plus sterile acupuncture needles, with copper handles, manufactured by HMD Europe London, UK. The needles were manually inserted by tapping the end of the copper handle, outside the guiding tube. The needles were manually manipulated by twirling, thrusting in and out after every 5 minutes till the patient experienced a similar sensation to that she experienced during initial manipulation, post-needle insertion.

In all the sessions, the physiotherapist was careful to avoid any accidents like needle injuries, stuck or bent needles. The physiotherapist was also well prepared, if these circumstances had arisen. All measures were taken to avoid any infections, such as washing hands with alcohol rub, avoidance of touching the needle's shafts, etc. No accidents or adverse reactions were reported during and post treatment.

Acupuncture session 1: The re-examination showed a reduction in lumbar spine AROM by $40 \%$ in all the movements. Mild pain was evident in the flexion of the lumbar spine. The patient was cautious while making movements. Apart from mild stiffness and tenderness, all other findings during re-examination were normal. After re-examination, the acupuncture treatment was carried out.

Before commencing acupuncture, the treatment site was examined for any skin or sensory problems, as these are contraindications for the treatment. After the pinprick sensation was experienced by the patient on needle insertion, the needle was manipulated by twirling, and thrusting in and out. Deqi was achieved, described as dull ache and mild tingling around the needle. The patient felt slightly drowsy post-treatment, which is a normal response.

Acupuncture session 2: The next follow-up session was carried out after 2 weeks. The patient reported soreness around the treatment area for 24 hours, but later experienced reduction in her symptoms. This improvement lasted for 6 or 7 days. The lumbar spine AROM was reduced in the bilateral side flexion, and mild pain and discomfort was evident in the extension and side flexion. There was mild tenderness on the lower lumbar spine. All the other objective markers were normal.
As the last acupuncture session was successful, the patient was comfortable and felt confident in continuing with the treatment. The deqi achieved during this session was comparatively stronger than the preceding session.

Acupuncture session 3: During the next follow-up, after 3 weeks, the patient reported experiencing no pain for 5 days after the session. The symptoms gradually came back after 6 or 7 days. There was improvement in the lumbar spine AROM, but patient remained cautious while moving, to avoid worsening the symptoms. There was no change in the lumbar spine palpation. Since the treatment was effective for the few days following treatment, the patient wished to continue.

Acupuncture session 4: During the next follow-up, after one week, the patient reported of further improvement in her symptoms. The lumbar spine AROM was full, and mild pulling in the lower back was evident in the flexion. Patient experienced pain, occasionally, during prolonged standing and bending. The patient was very pleased by the reduction in symptoms by acupuncture and wanted to continue with further treatment.

However, acupuncture could not be carried out during this session as patient was going to work post-session, and she did not wish to be drowsy. Since this was the last session, the patient asked the physiotherapist to request the GP for re-referral for more acupuncture sessions. The physiotherapist discharged the patient, and complied with the patient's request for re-referral from the GP.

\section{Clinical reasoning for the acupuncture points selected}

Bladder (BL) 23 (Shenshu), BL 24 (Qihaishu), BL 25 (Dachangshu): These points were selected to produce a localised effect, by releasing the inflammatory exudate such as bradykinin, potassium ions, histamine, and serotonin. This exudate causes vasodilation, leading to vascular permeability [21].

3 HJJ (Huatuojiaji): These points were added to enhance the local effect.

LI 4 (Hegu): With general musculoskeletal pain, including chronic LBP, this point has a general analgesic effect [22].

All these points were also selected for producing a segmental analgesic effect by stimulating $\mathrm{A} \delta$ fibres in the skin, and type 2 and 3 in muscle. This will block nociceptive $C$ fibre input by producing enkephalin, via intermediate cell activation [23].

\section{Discussion}

The current case report shows the short term effectiveness of acupuncture for chronic mechanical LBP, and therefore supports previous evidence [15]. However, even though this study has demonstrated its effectiveness, it is difficult to state 
affirmatively that acupuncture is the best treatment for chronic LBP. This is due to various factors involved: the first is the lack of the continuity of the treatment in the current study. Though the patient was informed that acupuncture will be beneficial if the sessions are carried out weekly, due to personal reasons, the patient could not attend the sessions regularly. Previous studies showing the effectiveness of the acupuncture on various musculoskeletal disorders involved frequent and regular sessions $[10,24]$. The current patient reported a gradual onset of LBP, which supports the evidence suggesting a favourable efficacy under similar circumstances [9]. Thus, if the patient had sought physiotherapy treatment in the early stages, acupuncture would have had a greater effect, as suggested by Karyannis et al. [7]. The improvement in the AROM of the lumbar spine can be due to the manual manipulation of the needle, which along with the de-qi effect caused the connective tissue to stretch. On removal of the needles, the tissue remained in the stretched position, causing the increase in AROM [25]. The Bladder and Huatuojiaji points would have caused this localised effect, since they are located near the spine. In addition, while many studies have demonstrated the positive results of acupuncture in cases where the patient insists on acupuncture as the only treatment for their symptoms, there is an opposing viewpoint, according to Thomas et al. [26] where an individual with an ambivalent attitude or expectation during acupuncture has shown to have had benefitted more. Hence the outcome of the current study could also be attributed to the young patient's initial sceptical attitude towards acupuncture.

The other factor, which could have assisted in extending the effect of the acupuncture in the current study, is the addition of acupuncture to other physical therapy interventions. Clinically, acupuncture in addition to manual therapy during the same session has shown to be effective in easing symptoms. Theoretically, manual therapy can be used before or after an acupuncture session, but clinically it has been observed that manual therapy before acupuncture is more effective. The difficulty lies in adding 2 or more physical therapy interventions in the span of one session, as the time allotted to NHS-referred patients is short. Since the patient was treated in a NHS clinical setting, the sessions lasted for 30 minutes each only. If they were in a private clinical setting, sessions could have been carried out for longer.

\section{Limitation/Further Research}

The findings of the current study should be considered with caution due to limitations involved as below:

- It is a case study involving only one patient. Further study involving a large sample size, long term follow up and with robust methodology is recommended.
- As mentioned previously, there was a lack of continuity in the treatment sessions, which is important for enhancing the efficacy of the acupuncture.

- There is a lack of use of more reliable and validated outcome measures, such as electro goniometer, which would have given an accurate reading of the lumbar spine AROM.

- There is a lack of functional improvement scale measurement. Though it is mentioned in the case report that there was a reduction in the pain during the aggravating factors but a functional improvement scale would have been a validating and reliable outcome measure.

- Although the case study helps in proving the efficacy of acupuncture in reducing the pain in a chronic low back pain patient but there is a lack of information related to VAS level improvement during each acupuncture sessions.

- Further research involving acupuncture with other physiotherapy interventions, like manual therapy, is recommended to facilitate the amalgamation of acupuncture as a treatment in various musculoskeletal disorders. Another aspect that can be investigated is the change in effect of the treatment, by changing the order of the interventions carried out.

- There was a lack of availability of electro-acupuncture (EA) modalities, which could have helped to provide higher stimulation. Various studies have shown the efficacy of EA over manual acupuncture [27].

\section{Conclusion}

The current case study has shown the short term efficacy of acupuncture as a treatment for a chronic LBP condition in reducing pain. Though it lacks a robust methodology, it does indicate the effectiveness of convention medical treatment in managing a musculoskeletal disorder, which assists in providing patient centred care. Since pain dramatically alters a patient's lifestyle and acupuncture helps in easing pain, it could eventually help the patient to return to their normal lifestyle [28].

Though the recent NICE guideline has excluded acupuncture from the non-invasive treatment section for LBP (NICE 2017), clinically acupuncture could still be beneficial in easing the symptoms. The exclusion of acupuncture from the guidelines can be more likely due to lack of good quality research in the field of the beneficial effect of acupuncture in LBP.

\section{Acknowledgement}

I would like to thank Mr Mike Pole-Baker for critically analysing and giving his feedbacks on each and every draft of this article. I would also like to thank Mr Paul Battersby for being an excellent Tutor and for his guidance and feedback during 
the foundation course of acupunture. And lastly, I would like to thank my family for always being supportive.

\section{References}

1. Deyo RA, Mirza SK, Martin BI (2006) Back pain prevalence and visit rates: estimates from US national surveys, 2002. Spine 31(23): 2724 2727.

2. Riksman JS, Williamson OD, Walker BF (2011) Delineating inflammatory and mechanical sub-types of low back pain: a pilot survey of fifty low back pain patients in a chiropractic setting. Chiropr Man Therap 19(1): 5.

3. Waddell G (1998) The back pain revolution. Churchill Livingstone, Edinburgh, UK, p. 438.

4. Vas J, Perea Milla E, Mendez C, Silva LC, Galante AH, et al. (2006) Efficacy and safety of acupuncture for the treatment of non-specific acute low back pain: a randomised controlled multicentre trial protocol [ISRCTN65814467]. BMC complementary and alternative medicine 6(1): 14.

5. Cheshire A, Polley M, Peters D, Ridge D (2013) Patient outcomes and experiences of an acupuncture and self-care service for persistent low back pain in the NHS: a mixed methods approach. BMC complementary and alternative medicine 13: 300 .

6. National Institute for Health and Clinical Excellence (NICE) (2009) Low back pain: early management of persistent non-specific low back pain. NICE Clinical Guideline 88. National Institute for Health and Clinical Excellence, London.

7. Karayannis NV, Jull GA, Hodges PW (2012) Physiotherapy movement based classification approaches to low back pain: comparison of subgroups through review and developer/expert survey. BMC Musculoskelet Disord 13(1): 24.

8. Witt CM, Jena S, Selim D, Brinkhaus B, Reinhold T, et al. (2006) Pragmatic randomized trial evaluating the clinical and economic effectiveness of acupuncture for chronic low back pain. Am J Epidemiol 164(5): 487-496.

9. Smedley J, Inskip H, Buckle P, Cooper C, Coggon D (2005) Epidemiological differences between back pain of sudden and gradual onset. J Rheumatol 32(3): 528-532.

10. Oakes H, Bassett P (2015) Group acupuncture versus individual targeted acupuncture for reducing pain in individuals with low back pain. Acupuncture in physiotherapy 27(2): 37-48.

11. Laslett M, McDonald B, Aprill CN, Tropp H, Őberg B (2006) Clinical predictors of screening lumbar zygapophyseal joint blocks: development of clinical prediction rules. Spine J 6(4): 370-379.

12. Hicks GE, Fritz JM, Delitto A, McGill SM (2005) Preliminary development of a clinical prediction rule for determining which patients with low back pain will respond to a stabilization exercise program. Arch Phys Med Rehabil 86(9): 1753-1762.
13. Flynn T, Fritz J, Whitman J, Wainner R, Magel J, et al. (2002) A clinical prediction rule for classifying patients with low back pain who demonstrate short-term improvement with spinal manipulation. Spine 27(24): 2835-2843.

14. Yun M, Shao Y, Zhang Y, He S, Xiong N, et al. (2012) Hegu acupuncture for chronic low-back pain: a randomized controlled trial. J Altern Complement Med 18(2): 130-136.

15. Manheimer E, White A, Berman B, Forys K, Ernst E (2005) Metaanalysis: acupuncture for low back pain. Ann Intern Med 142(8): 651663.

16. Refshauge K, Gass E (2004) Musculoskeletal physiotherapy: clinical science and evidence-based practice, Oxford: ButterworthHeinemann, UK

17. Szadek KM, vander Wurff P, van Tulder MW, Zuurmond WW, Perez RS (2009) Diagnostic validity of criteria for sacroiliac joint pain: a systematic review. J Pain 10(4): 354-368

18. Laslett M, Aprill CN, McDonald B, Young SB (2005) Diagnosis of sacroiliac joint pain: validity of individual provocation tests and composites of tests. Man Ther 10(3): 207-218.

19. Sivayogam A, Banerje A (2011) Diagnostic performance of clinical tests for sacroiliac joint pain. Physical Therapy Reviews Journal 16(6): 462467.

20.van Trijffel E, Anderegg Q Bossuyt PMM, Lucas C (2005) Interexaminer reliability of passive assessment of intervertebral motion in the cervical and lumbar spine: a systematic review. Man Ther 10(4): 256-269.

21.Sandberg M, Lundeberg T, Lindberg LG, Gerdle B (2003) Effects of acupuncture on skin and muscle blood flow in healthy subjects. Eur J Appl Physiol 90(1-2): 114-119.

22. Lian YL, Chen CY, Hammes M, Kolster BC (2005) Pictorial Atlas of acupuncture: an illustrated manual of acupuncture points. H. F Ullmann, Cologne, Germany.

23. White A, Cummings M, Filshle J (2008) An Introduction to Western Medical Acupuncture. Churchill Livingstone, UK.

24. Gay S (2015) Acupuncture for chronic bilateral ankle osteoarthritis in a 60-year-old male. Acupuncture in physiotherapy 27(1): 79-85.

25. Langevin HM (2013) The science of stretch

26. Thomas KJ, MacPherson H, Thorpe L, Brazier J, Fitter M, et al (2006) Randomised controlled trial of a short course of traditional acupuncture compared with usual care for persistent non-specific low back pain. BMJ 333(7569): 623 .

27. Wang SM, Kain ZN, White P (2008) Acupuncture analgesia: I. The scientific basis. Anesth Analg 106(2): 602-610.

28. Farrar JT, Young JP, LaMoreaux L, Werth JL, Poole RM (2001) Clinical importance of changes in chronic pain intensity measured on an 11-point numerical pain rating scale. Pain 94(2): 149-158. 
This work is licensed under Creative Commons Attribution 4.0 License

DOI: 10.19080/JYP.2018.03.5555618
Your next submission with Juniper Publishers will reach you the below assets

- Quality Editorial service

- Swift Peer Review

- Reprints availability

- E-prints Service

- Manuscript Podcast for convenient understanding

- Global attainment for your research

- Manuscript accessibility in different formats

( Pdf, E-pub, Full Text, Audio)

- Unceasing customer service

Track the below URL for one-step submission https://juniperpublishers.com/online-submission.php 